\title{
Detecting Unknown Persons using Survillance Camera
}

\author{
P.C.Karthik, M.Chandra, D.Jagadeesh
}

\begin{abstract}
The security is a situation where items, creatures or individuals are given special identifiers and the capacity to move information over a system without expecting human-to-human or human-to-PC cooperation. The webcam has developed from the assembly of remote innovations and the Internet. The security framework is the correspondence of anything with some other thing, the correspondence for the most part moving of useable information, for instance, a sensor in a space to screen and control the temperature. To depict a security alert framework utilizing low handling force chips utilizing Internet of things which assists with checking and get cautions when movement is distinguished and sends the data to approved individual. In addition, Internet of things based application can be utilized remotely to see the action and get notices when movement is distinguished. Hence, focal points like these make this application perfect for checking homes in nonappearance.
\end{abstract}

Keywords: human-to-human or human-to-PC.

\section{INTRODUCTION}

Video reconnaissance framework has become a basic part in the security and insurance arrangement of present day urban areas, since shrewd checking cameras furnished with savvy video examination systems can screen and pre-alert strange practices or occasions. In any case, with the extension of the observation organize, monstrous reconnaissance video information presents gigantic difficulties to the investigation, stockpiling and recovery in the Big Data period. This task introduces a novel savvy preparing and use answer for huge observation video information dependent on the occasion location and disturbing messages from front-end shrewd cameras. The technique incorporates three sections: the observation video examination and picture catch, Anomaly recognition and smart pre-disturbing for strange occasions. Brilliant stockpiling for reconnaissance video and fast recovery for proof recordings, which completely investigates the transient spatial affiliation examination regarding the anomalous occasions in various checking destinations. Test results uncover that our proposed approach can dependably pre-alert security chance occasions, generously lessen extra room of recorded video and essentially accelerate the proof video recovery related with explicit suspects.

Present day urban areas are normally presented to crisis circumstances, for example, car crashes, fear based oppressor assaults and wrongdoings.

Revised Manuscript Received on April 25, 2020.

* Correspondence Author

P.C.Karthik*, Department of CSE, SRM Institute Of Science, and Technology, Kattankulatur,India karthikc@srmist.edu.in

M.Chandra, Department of CSE, SRM Institute Of Science, and Technology, Kattankulatur,India marthalachandra15@gmail.com

D.Jagadeesh, Department of CSE, SRM Institute Of Science, and Technology, Kattankulatur,India jagadeesh.cd@gmail.com

(C) The Authors. Published by Blue Eyes Intelligence Engineering and Sciences Publication (BEIESP). This is an open access article under the CC BY-NC-ND license (http://creativecommons.org/licenses/by-nc-nd/4.0/)
As a regular model, Paris fear assaults in 2015 remaining at any rate 129 individuals dead. So as to stop lawbreakers and limit standardized savings risks, countless savvy checking cameras and reconnaissance frameworks have been broadly conveyed in urban regions. The recorded voice and video information are valuable for researching situations when wrongdoings really occur. Video reconnaissance frameworks have been playing an ever increasing number of basic jobs in wrongdoing anticipation and legal. Driven by video examination and Big Data methods as of late, insightful video reconnaissance frameworks have seen fast advancement, jumping up an enormous number of delegate specialized accomplishments and applications. In the part of capacity and recovery for Big Data, Guo et al. planned a strategy named SVIS to spare video information for resulting investigation of working plat-structures. Shavachko et al. made HDFS framework to spare enormous video information viably so as to determine the issue that capacity excess and huge space utilization are brought about by video information. Huang et al. built a recovery systematical technique to improve the effectiveness and exactness of recovery brings about the Big Data period. Dey et al. utilized cloud information store and built up an interactive media reconnaissance backend framework dependent on the Sensor Web Enablement structure. They gathered video information that originates from various observation cameras, split media documents and metadata and put away those in an isolated manner in cloud, which understands a progressively sensible administration and increasingly fast recovery. Ding et al. proposed a novel system for enormous scale cross-methodology search by means of aggregate grid factorization hashing, which changes information into double portrayal to perform fast hunt with low stockpiling cost. Xiao et al. abused worldwide repetition in huge observation video information of numerous sources in enormous time range for proficient stockpiling. Be-sides, with the assistance of distributed storage, an enormous number of capacity systems have been created for large information stockpiling. The accompanying points of interest of our new detailing of video occasion location:

1. By finding the ideal spatiotemporal way, our strategy decides the beginning and the finish of the video occasion consequently, and can definitely limit the occasion in every video outline. It is powerful to the bogus and missed nearby recognitions and along these lines can successfully deal with overwhelming impediments. 
2. As both positive and negative preparing models are used for a discriminative preparing, our technique is vigorous to intraclass varieties of the video occasions and the jumbled and dynamic foundations.

3. Our proposed strategy can be effectively reached out to deal with scale and shape varieties of the occasion, and can identify various occasions at the same time.

We have proposed a novel methodology for distinguishing perplexing and dynamic occasions. The unwinding from video sub volume to spatiotemporal way makes the technique progressively adaptable and, henceforth, very much routed to complex occasions that couldn't be decisively secured by sub volume. The worldwide ideal arrangement of our Max-Path calculation improves the smoothness of the occasion, hence killing the bogus positives and mitigates missed or frail discoveries because of impediments and the picture low quality. What's more, Max-Path's most minimal unpredictability makes it proficient to scan for spatiotemporal ways in an enormous 5D space of spatiotemporal, scale, and shape. At last, our investigations on three unique kinds of occasions, utilizing various highlights, with both nearby and worldwide article portrayals demonstrated that our proposed technique is general and adaptable enough to be applied to a wide class of occasions.

\section{RELATED WORK}

Writing Survey is the most significant advance in Software Development process. Prior to building up the apparatus, it is important to decide the time factor, economy and friends quality. When these things are fulfilled, at that point subsequent stages are to figure out which working framework and language can be utilized for building up the instrument. When the developers begin constructing the apparatus the software engineers need part of outside help. This help can be acquired from senior developers, from book or from sites. Before building the framework the above thought is considered for building up the proposed framework.

It's a major test to manage security in a city. Innovation progressions are impacting our life, urban areas are developing, and present day urban communities are alluding increasingly more to computerized advances. At present, a tremendous measure of independent autonomous frameworks works in the city, they will probably fulfill some business exercises, for example banking, customs, medical clinics, and so on. Information gathered by these frameworks speaks to, whenever incorporated, a key component in any basic leadership process. This paper exhibits a, working, shrewd synergistic stage to incorporate different frameworks to serve the observation exercises in a city or nation. It comprises of a community oriented reconnaissance framework, called CityPro. The engineering that we propose is a future vision to ensure individuals and screen open frameworks, for example, spans, streets, structures, and so forth.; it is intended to manage as well as forestall irregular exercises like fear monger assaults. CityPro is required to work in live-mode by utilizing (planned to utilize) city adjusted ITframeworks. Toward the finish of this paper, a run of the mill contextual analysis is given, and difficulties and future works are likewise examined Albeit sliding window-based methodologies have been very effective in identifying objects in pictures, it's anything but an insignificant issue to extend them to recognizing occasions in recordings. We propose to scan for spatiotemporal ways for video occasion location. This new definition can precisely recognize and find video occasions in jumbled and swarmed scenes, and is strong to camera movements. It can likewise well deal with the scale, shape, and intraclass varieties of the occasion. Contrasted with occasion identification utilizing spatiotemporal sliding windows, the spatiotemporal ways relate to the occasion directions in the video space, therefore can all the more likely handle occasions created by moving items. We demonstrate that the proposed search calculation can accomplish the worldwide ideal arrangement with the most minimal unpredictability. Analyses are led on practical video informational indexes with various occasion identification assignments, for example, peculiarity occasion location, strolling individual discovery, and running recognition. Our proposed technique is good with various sorts of video highlights or article locators and powerful to bogus and missed neighborhood identifications. It altogether improves the general discovery and confinement precision over the cutting edge strategies.

\section{SYSTEM ANALYSIS}

\subsection{Existing System}

- $\quad$ The existing shrewd observation frameworks video recorded and checking through PC or savvy TV.

- It won't cozy or alarm if any unusual condition happens and it requires manual intercession moreover.

- However, it is very not convincible to pass judgment on suspicious conduct by a solitary checking.

- It possibly bodes well to regard the meandering as doubt when it happens over and again or takes quite a while.

\section{Disadvantages}

- No alert system.

- Lot of data storage which increase cost.

- Manual monitoring has be done all the time.

- $\quad$ Easy access or block using jammer.

\subsection{Proposed System}

- This ventures manages the plan and usage of Smart reconnaissance observing framework utilizing Raspberry pi.

- Advocates an astute handling way to deal with huge reconnaissance video information driven by cameras.

- We use camera to catch and break down the information in any event, for a small scale sec to accomplish high security in home.

- It's catching the picture and contrast it and past picture through K-closest neighbor calculation and Decision tree calculation.

\section{Advantages}

- It offers unwavering quality.

- Immediate ready framework.

- We will have the option to alarm the individuals through mail utilizing IOT innovation. 


\section{BLOCKDIAGRAM}



- This ventures manages the structure and usage of Smart reconnaissance checking framework utilizing Raspberry pi.

- Advocates a savvy preparing way to deal with huge observation video information driven by cameras.

- We use camera to catch and break down the information in any event, for a miniaturized scale sec to accomplish high security in home.

- It's catching the picture and contrast it and past picture through K-closest neighbor calculation and furthermore we actualize mapping and information decrease utilizing map lessen calculation.

- $\quad$ Raspberry pi is a Visa measured PC. It works nearly as a PC. There are different reconnaissance frameworks, for example, camera, CCTV and so on.,

- In these kinds of reconnaissance frameworks, the individual who is stationary and is situated in that specific territory can just view what's going on in that spot.

- Whereas, here, regardless of whether the client is moving starting with one spot then onto the next, he/she can monitor what's going on in that specific spot. Additionally another preferred position is that it offers protection on the two sides since it is being seen by just a single individual.

- The other significant bit of leeway is that it is a basic circuit. The working framework utilized here is Raspbian OS.

\section{MODULES DESCRIPTION}

\section{Video Analysis}

The keen video observation framework utilizes 3-level engineering that contains customer side, application server and a database server. The application server comprises of the server gadget which is outfitted with a STMP Mail Service. The customer side gadget needs just the program. It will associate with server through IP address and port number. The server side gadget chiefly contains two modules one is http server and another is picture preparing. Two gadgets speak with one another with http convention. Thus http server is required. It will work in server side gadget itself. The fundamental working of this is to deal with the up and coming solicitations, check for approval and produce the reaction. The picture handling module is to recognize interruption.

\section{Image Processing}

The SSE is based on the following key video analysis Technologies:

Object Detection: This arrangement of advancements can identify moving articles in a video grouping produced by a static camera. The recognition procedures are tolerant to changes in characteristic lighting, sensible changes in the climate, diverting developments (like trees waving in the breeze), and camera shake.

Object Tracking: This arrangement of advancements can follow the shape and position of numerous articles as they move around a space that is checked by a static camera. The strategies are intended to deal with huge impediments as items interface with each other.

Object Classification: These advances utilize different properties of an item including shape, size and development to dole out a class name to the articles. Run of the mill names incorporate, Person, Group and Vehicle.

\section{Voice Alert}

The caution data of unordinary practices, which is caught by front-end brilliant cameras, gave to develop the irregular conduct database. The anomalous conduct database will give the metadata to the consequent investigation. The unmistakable data gave by metadata incorporates the kind of checking site and strange conduct, name of observing site, ID of shrewd camera, time of unusual conduct happening, time of irregular conduct finishing, record of observation video, depiction or video clasp of related conduct.

\section{Mail Service}

The client gets told when the interruption is identified. Hence, the client can make proper move immediately. Shrewd video observation is incorporated with insightful video development location examination frameworks consolidate with Mail warning framework. In outline, by a mix of depiction pictures, unique reconnaissance recordings and uncommon occasions, significant hints can be discovered a lot simpler. What's more, get a shrewd disturbing for strange occasions.

\section{CONCLUSION}

Rather than the standard video perception system, the proposed course of action adds to use recognized and alarmed events by splendid checking cameras, which right now improves the introduction of insightful surveillance structure, hoists the ability to hazard pre-upsetting, and hugely saves the additional space for perception video data. At that point, the surveillance video data essential to express cases will be cut back, which will fundamentally improve the capability for finding noteworthy assessment snippets of data. A couple of rational cases show that our approach outmanoeuvres the present courses of action.

\section{FUTURE WORK}

Therefore, the ingested data will have needed designs (for instance sorted out data, well-encoded video game plan records) and thus can be destitute down authentically. With a significantly flexible plan and a sharp schedule engine, SVIS can be dynamically scaled out to manage tremendous scale online camera streams and raised ingestion occupations. SVIS is in like manner especially extendable. It describes various interfaces to enable introducing customer portrayed modules to help new sorts of video source and data sink.

Published By:

Blue Eyes Intelligence Engineering \& Sciences Publication 
Exploratory results show that SVIS system has high adequacy and extraordinary adaptability.

The stage 2 of this endeavor is to realize the mail correspondence module where the customer will get told with the strange lead captured as an image through mail when it got occurred. Furthermore, viability and accuracy are the basic concerns when the system is being executed dynamically. So Various estimations have been used to test the system execution on its throughput.

\section{REFERENCES}

1. X. Guo, Y. Cao, and J. Tao, "SVIS: Large Scale Video Data Ingestion into Big Data Platform," Database Systems for Advanced Applications, DASFAA 9052, A. Liu et al., eds., Springer, 2015, pp. 300 - 306.

2. S. Konstantin, H. Kuang, R. Sanjay, and R. Chansler, "The Hadoop Distributed File System," in Proc. IEEE Symposium on Mass Storage Systems and Technologies (MSST), 2010, pp. 110

3. Y. Huang, J. Schuehle, A. L. Porter, and J. Youtie, "A Systematic Method to Create Search Strategies for Emerg ing Technologies Based on the Web of Science: Illustrated for „Big Data ${ }^{e e}, "$ Scientometrics, vol. 105, no. 3, pp. 2005- 2022, 2015.

4. D. Swarnava, C. Ankur, N. Soumitra, and M. Prateep, "Smart City Surveillance Leveraging Benefits of Cloud Data Stores," in Proc. IEEE Conference onLocal Computer Networks Workshops (LCN Workshops), 2012, pp. 868- 876.

5. M. Dbouk, H. McHeick, and I. Sbeity, "CityPro; An Integrated City-protection Collaborative Platform,"Procedia Computer Science, vol. 37, pp. 72-79, 2014.

6. P. Ji, Y. Kim, Y. Yang, and Y.-S. Kim, "Face Occlusion Detection Using Skin Color Ratio and LBP Features for Intelligent Video Surveillance Systems," in Proc. Federated Conference on Computer Scienceand Infor-mation System (FedCSIS), 2016, pp. 253-259.

7. M.-C. Tuan and S.-L. Chen, "Fully Pipelined VLSI Architecture of a Real-time Block-based Object Detector for Intelligent Video Surveillance Systems," in Proc. IEEE/ACIS 14th International Conference on Computer and Information Science (ICIS), 2015, pp. 149-154.

8. J. Ju, B. Ku, D. Kim, T. Song, D. K. Han, and H. Ko, "Online Multi-person Tracking for Intelligent Video Surveillance System," in Proc. IEEE International Conference on Consumer Electronics (ICCE), 2015, pp. 345-346.

9. X. Guo, Y. Cao, and J. Tao, "SVIS: Large Scale Video Data Ingestion into Big Data Platform," Database Systems for Advanced Applications, DASFAA 9052, A.Liu et al., eds. Springer, 2015, pp. 300-306.

10. J. Xiao, L. Liao, J. Hu, Y. Chen, and R. Hu, "Exploiting Global Redun-dancy in Big Surveillance Video Data for Efficient Coding," Cluster Computing, vol. 18, pp. 531-540, 2015

11. H. Kim, S. Lee, Y. Kim, S. Lee, D. Lee, J. Ju, et al., "Weighted Joint-based Human Behavior Recognition Algorithm Using only Depth Infor-mation for Low-cost Intelligent Video-surveillance System,"Expert Sys-tems with Applications, vol. 45, pp. 131141, 2016.

12. O. Boiman and M. Irani, "Detecting Irregularities in Images and in Video," International Journal of Computer Vision,vol. 74, pp. 17-31, 2007.

13. C. Chen, Y. Shao, and X. Bi, "Detection of Anomalous Crowd Behavior Based on the Acceleration Feature," IEEE Sensors Journal, vol. 15,no. 12,pp. 7252-7261, 2015.

14. Y. Cong, J. Yuan, and J. Liu, "Sparse Reconstruction Cost for Abnormal Event Detection," in Proc. IEEE Conference on Computer Vision and Pattern Recognition (CVPR), 2011, pp. 3449-3456. 